\title{
Imaging and Analysis of THz Surface Plasmon Polariton Waves with the Integrated Sub-wavelength Aperture Probe
}

\author{
Raimund Mueckstein • Chris Graham • \\ Cyril C. Renaud • Alwyn J. Seeds • \\ James A. Harrington • Oleg Mitrofanov
}

Received: 30 December 2010 / Accepted: 18 July 2011

(C) Springer Science+Business Media, LLC 2011

\begin{abstract}
We demonstrate that the integrated sub-wavelength aperture probe designed for $\mathrm{THz}$ near-field scanning probe microscopy can be used to map surface plasmon waves at $\mathrm{THz}$ frequencies. Observed near-field images of metallic patterns reveal surface plasmon waves superimposed over $\mathrm{THz}$ transmission images. We discuss the coupling mechanism for the surface waves and arrive to an important conclusion that the detected surface wave images represent the spatial derivative of the surface plasmon electric field. The relationship between the electric field and the measured signal is confirmed experimentally by mapping surface waves in bow-tie antennas. This study explains previously observed effects in $\mathrm{THz}$ near-field microscopy and provides a framework for analysis of near-field images.
\end{abstract}

Keywords Terahertz $\cdot$ Near-field $\cdot$ Surface waves $\cdot$ Surface plasmon polariton $\cdot$ Terahertz imaging $\cdot$ Antenna $\cdot$ Near-field probe $\cdot$ Sub-wavelength aperture

\section{Introduction}

Terahertz (THz) surface plasmon polaritons (SPP), also known as surface waves, provide a useful possibility to guide and confine the electromagnetic energy near metallic surfaces without radiation [1-8]. Surface waves are in the core of many interesting phenomena, such as the extraordinary transmission of sub-wavelength hole arrays, the second harmonic generation in metals, and the negative value of the refractive index in metamaterials. Visualization of SPP waves has been critical for recognizing their role in physical phenomena [9-12]. However the non-radiative nature of SPP waves results in difficulties

R. Mueckstein · C. Graham • C. C. Renaud • A. J. Seeds • O. Mitrofanov $(\bowtie)$

Department of Electronic and Electrical Engineering, University College London, Torrington Place, London WC1E 7JE, UK

e-mail: o.mitrofanov@ucl.ac.uk

\section{J. A. Harrington}

Department of Materials Science and Engineering, Rutgers University, 607 Taylor Rd., Piscataway, NJ 08854, USA 
for their experimental investigations: coupling to the free-space propagating waves is weak and detection of SPP waves is in general destructive.

Detection of surface waves is a particularly difficult problem and it requires special probes. At $\mathrm{THz}$ frequencies, several near-field microscopy probes can be used to map and investigate surface waves. The electro-optic THz probes, for example, provide a remarkable possibility to detect and differentiate between individual components of the electric field vector $[12,13]$; the scattering near-field probes, such as a sharp metallic needle, allow mapping of the THz field with an unprecedented spatial resolution [14]. The integrated subwavelength aperture near-field probe [15] was also recently applied to study $\mathrm{THz}$ SPP waves. We mapped a previously undetected phenomenon with this probe, a surface wave formed on a metallic surface by a tightly focused $\mathrm{THz}$ beam [16].

Detection of SPPs with this probe is not obvious because the detector antenna in the probe is orthogonal to the polarization of the SPP field. Nevertheless, the surface nature of SPP waves allows them to couple through the probe aperture and to induce a current in the photoconductive antenna detector. The application of the integrated sub-wavelength aperture near-field probe for $\mathrm{THz}$ SPP studies opens exciting opportunities because the probe response covers a broad band of frequencies and it allows imaging $\mathrm{THz}$ fields with a spatial resolution better than 10 microns [15]. This paper is focused on exploring possibilities for SPP imaging with the integrated sub-wavelength aperture probe. We explain the coupling mechanism that allows detection of surface waves and illustrate the probe capabilities by mapping SPP waves in several experiments: excitation of surface waves near the focus of a $\mathrm{THz}$ beam and at the edges of a metallic object. We also demonstrate how the near-field probe can be applied for studies of SPP resonances in a $\mathrm{THz}$ bow-tie antenna. These experimental investigations confirm a very important property of the probe, which follows from the developed coupling mechanism. Specifically, the integrated sub-wavelength aperture probe detects a spatial derivative of the SPP field rather than the field itself.

The understanding of the SPP coupling mechanism provides a framework for analysis and interpretation of images in near-field microscopy. The coupling mechanism also explains puzzling effects that have been observed in earlier near-field microscopy measurements, such as the appearance of bright features behind opaque metallic objects. This imaging method can provide a vital insight into the $\mathrm{THz}$ SPP role in basic physical phenomena and $\mathrm{THz}$ devices. It can also help determining experimental values for SPP characteristics.

\section{Detection of SPP waves: coupling mechanism}

The coupling of the SPP waves into the sub-wavelength aperture probe is different from the coupling of the free-space propagating waves. For the SPP wave, the polarization of the electric field is typically normal to the aperture plane, whereas for the free-space waves, the polarization of the incident field is predominantly in the aperture plane. The difference in the polarization results in a different relationship between the detected signal and the electric field that causes it. In the case of the surface wave, the detected signal represents a spatial derivative of the SPP field rather than the actual field. The mechanism that explains this relationship was proposed in [16]; here we briefly overview this mechanism and discuss its implications for near-field microscopy.

The sensitivity of the integrated sub-wavelength aperture probe to SPP waves can be explained by considering how a surface wave travelling along the top surface of a metallic 
film, can couple into a small aperture in the film. The coupling mechanism is schematically illustrated in Fig. 1. For the aperture size much smaller than the wavelength, the wave propagates along the surface without distortion due to the aperture. However, near the aperture, the distribution of the normal component of the electric field, $E^{S P P}$, and the corresponding charge density, becomes discontinuous. As a result, there will be an electric potential difference between the opposite edges of the aperture caused by the spatial variation of the SPP field $E^{S P P}(\vec{r})=E_{0} \exp \left(i \vec{k}_{S P P} \cdot \vec{r}\right)$, where $\vec{k}_{S P P}$ is the wave vector. The potential difference produces an in-plane electric field component $E_{t}$ across the aperture polarized in the direction of the surface wave propagation (Fig. 1b). It is this field, $E_{t}$, that is detected by the integrated sub-wavelength aperture probe, in which a photoconductive (PC) antenna is placed below the metallic film in the near-field zone of the aperture.

The field $E_{t}$ is induced entirely by the surface wave and it is proportional to the difference in the normal component of the SPP wave at the opposite edges of the aperture. In other words, $E_{t}$ is determined by the in-plane gradient of the instantaneous surface field $\nabla E^{S P P}(x, y)[16]$. The photocurrent in the antenna due to the surface wave, $i_{S P P}$, is therefore proportional to the projection of the gradient vector on the antenna vector $\vec{a}$, as illustrated in Fig. 1b for the case of a circular aperture. The relationship between the detected current and the surface field $E^{S P P}$ can be approximated by the following equation:

$$
i_{S P P}(x, y)=g_{1} \nabla E^{S P P}(x, y) \cdot \vec{a}=g_{1}\left(\vec{x} \frac{d}{d x} E^{S P P}(x, y)+\vec{y} \frac{d}{d y} E^{S P P}(x, y)\right) \cdot \vec{a},
$$

where $g_{1}$ is a constant, which represents a product of the aperture transmission coefficient and the PC antenna response. This equation describes the detected current in the subwavelength aperture probe due to the SPP wave travelling on its surface. A similar coupling mechanism applies in the near-field microscopy experiments, where SPP waves can exist between metallic surfaces of samples and the metallic surface of the near-field probe.

The fact that the current detected by the PC antenna is proportional to the in-plane spatial derivative of the SPP field $E^{S P P}$, rather than the actual field, has important implications for near-field microscopy: the detected signal depends not only on the strength of the SPP field, but also on the direction of wave propagation. This effect will be illustrated later in the discussion of experimental results. At this point we only note that due to the harmonic behaviour of the SPP wave in the plane of propagation, $E^{S P P}(x, y)=E_{0}(x, y) \exp \left(i k_{x, S P P} x+i k_{y, S P P} y\right)$, the spatial derivative nature of the detected current produces a spatial phase shift between the antenna current distribution $i_{S P P}(x, y)$ and
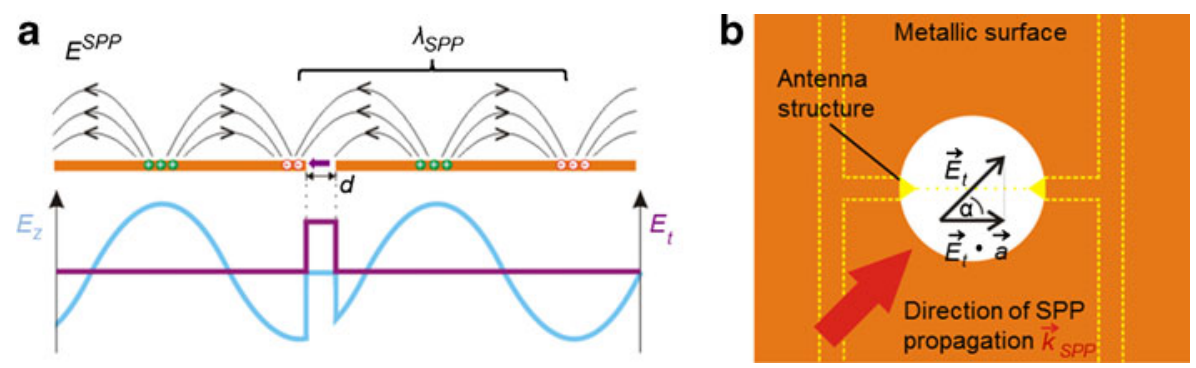

Fig. 1 a A schematic diagram of the surface wave coupling into a sub-wavelength aperture in the metallic film. b A top view of the integrated near-field probe; showing the photoconductive antenna detects and the in-plane electric field $E_{t}$ induced by the surface wave. 
the actual electric field distribution $E^{S P P}(x, y)$. For example, a relatively weak spatial variation of the SPP wave amplitude $E_{0}(x, y)$ results in a phase shift of approximately $\pi / 2$.

It is important to keep in mind that the total current in the probe antenna $i_{A}$ can also contain a contribution due to the $\mathrm{THz}$ field with the electric field vector polarized in the plane of the aperture. In a typical near-field microscopy system, the THz field with such polarization is used for sample illumination. Our earlier studies showed that this contribution can be approximated by the temporal derivative of the transverse incident field $E_{\text {inc }}(t)$ [17]. Therefore the total detected signal is a sum of two terms, one is due to the incident $\mathrm{THz}$ field $E_{\text {inc }}$ polarized in the aperture plane and the other is due to the surface wave field $E^{S P P}$, polarized perpendicular to the plane:

$$
i_{A}(x, y, t)=g_{1}\left(\vec{x} \frac{d}{d x} E^{S P P}(x, y, t)+\vec{y} \frac{d}{d y} E^{S P P}(x, y, t)\right) \cdot \vec{a}+g_{2} \frac{d}{d t} \vec{E}_{\text {inc }}(x, y, t) \cdot \vec{a} .
$$

where $g_{2}$, similarly to $g_{1}$, represents the aperture transmission properties for the transverse incident field and the PC antenna response. For our near-field probes, both terms in Eq. 2 produce comparable contributions to the detected current.

It is useful to consider how the surface plasmon waves can be excited in near-field microscopy measurements. In the collection mode microscope, a sample is typically illuminated uniformly through the substrate. The near-field probe is scanned over the sample surface as illustrated in Fig. 2. In this arrangement, the polarization of the incident field $E_{\text {inc }}$ is in the plane of the aperture and no surface wave is excited. However if the sample contains a metallic feature, for example an antenna patch or a transmission line, surface waves can be excited at the edges of this feature. In addition, it is also possible to excite a surface wave directly on the metallic surface of the probe by localized electric fields created by sharp features of the sample. The excited SPP wave in this case is carried by the metallic surface of the probe rather than the sample. Both types of the surface waves can appear in the near-field microscopy images as it will be shown later.

Equation 2 gives the vital information for interpretation of near-field images measured with the integrated sub-wavelength aperture probe. The equation describes contributions due to SPP waves and due to the illumination field. For correct image interpretation these
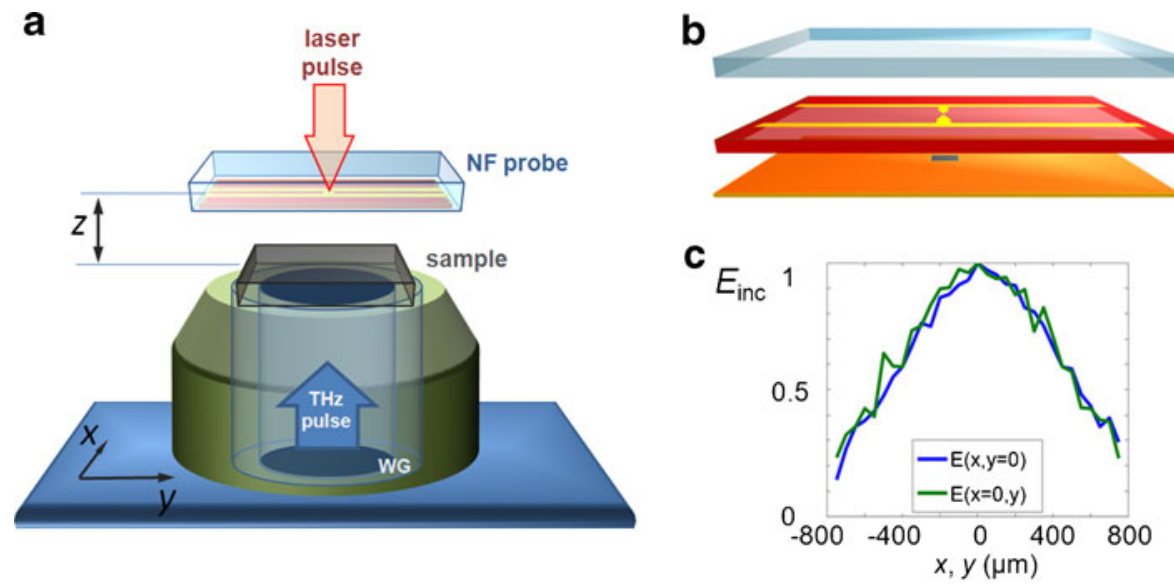

Fig. 2 a A schematic diagrams of the experimental setup for near-field imaging of surface plasmon waves. b elements of the integrated near-field probe shown separately. c The electric field amplitude profiles for the illuminating $\mathrm{THz}$ beam along the $x$ - and $y$-axis in the sample plane. 
contributions however must be distinguished. The time-domain analysis is very useful in this task as the surface waves have a distinctive signature in the space-time map [16]. It's also useful to compare images for different polarizations of the incident field and to utilize the discriminating property of the detector antenna. In some cases, the last term in Eq. 2 can be excluded completely when the incident field is polarized orthogonally to the antenna of the near-field probe. For these special cases, the original surface wave pattern $E^{S P P}(x, y)$ can be reconstructed by applying integration to the recorded near-field images. In the following examples we will apply our understanding of the coupling mechanism for analysis of near-field images.

\section{Imaging of SPP waves}

The experimental setup for mapping of SPP waves is shown in Fig. 2a. The setup is similar to the collection mode near-field scanning probe microscopy system $[15,16]$. The probe consists of a low-temperature grown GaAs PC antenna integrated between a sapphire substrate and a thin layer of GaAs $(\sim 5 \mu \mathrm{m})$, covered with a $600 \mathrm{~nm}$ gold film everywhere except for a small square aperture of size $d$ in the region of the dipole antenna (Fig. 2b) [18]. The PC antenna is gated by $100 \mathrm{fs}$ optical pulses from the Ti:Sapphire laser as in a standard $\mathrm{THz}$ time-domain spectroscopy setup. $\mathrm{THz}$ pulses are delivered to samples through a hollow core dielectric-lined cylindrical metallic waveguide [19, 20]. This waveguide provides a linearly polarized beam of a circular shape for uniform sample illumination (Fig. 2c). The waveform of the THz pulse contains approximately 3-4 cycles at $\sim 2 \mathrm{THz}$ due to dispersion in the waveguide [21, 22]. Samples are mounted on the $\mathrm{THz}$ waveguide and the sample-waveguide assembly is raster scanned with respect to the stationary probe to record near-field images (Fig. 2a).

For simplicity we define a coordinate system with the $x$-axis pointing in the direction parallel to the dipole antenna $\vec{a}$. In this case, only one spatial derivative survives in Eqs. 1 and 2. The incident $\mathrm{THz}$ beam is typically polarized in the $x$-direction to maximize the detected signal. However, the polarization of the incident $\mathrm{THz}$ beam can be rotated to a desired angle without changing position of the sample with respect to the probe. Alternatively, the sample-waveguide assembly can be rotated around the waveguide axis without affecting the polarization of the incident beam. The possibility to vary orientation of the sample and the incident beam polarization allows minimizing the contributions due to one of the spatial derivatives or the temporal derivative in Eq. 2.

\subsection{Surface waves due to the focused beam}

We first consider a surface wave excited by a focused $\mathrm{THz}$ beam on the metallic surface. In this experiment the sample in Fig. 2a is replaced by a high numerical aperture hyperhemispherical Si lens (1 mm radius), which focuses the $\mathrm{THz}$ beam to a $60 \mu \mathrm{m}$ diameter spot on the gold surface of a near-field probe with the aperture size $d=20 \mu \mathrm{m}$. A schematic diagram of this experimental setup is shown in Fig. 3a.

The focused beam contains a small longitudinal component of the electric field, $E_{z}$, which excites a surface plasmon wave on the near-field probe surface [16, 23-25]. The wave then diverges radially along the surface [25]. The focused incident field and the SPP wave are mapped in the $x y$-plane by scanning the lens-waveguide assembly with respect to the probe aperture (Fig. 3b). A dark spot in the middle of the map corresponds to the focused transverse $\mathrm{THz}$ beam. Concentric fringes around the central spot correspond to the SPP wave formed near the focal point and emanating in the $+x$ and $-x$ directions. This 
a

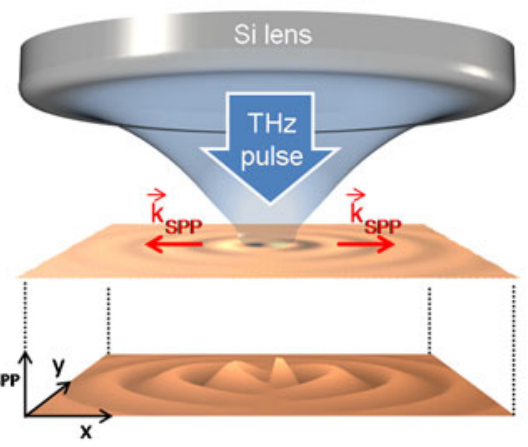

b

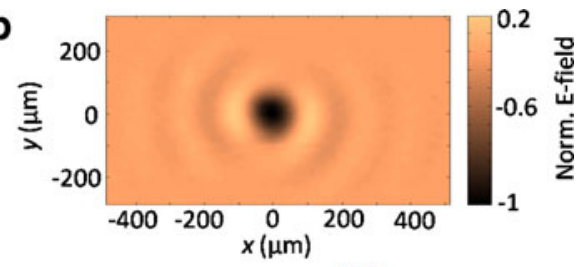

C

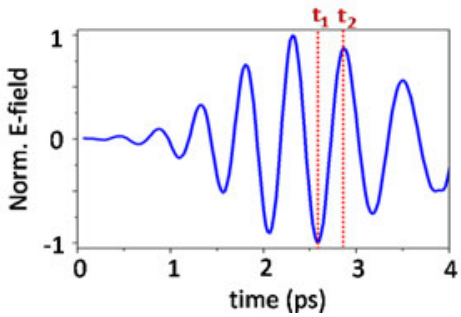

Fig. 3 a A schematic diagram of the surface wave excitation by a $\mathrm{THz}$ beam focused by a hyperhemispherical Si lens. b An image measured by the near-field probe ( $d=20$ micron) in the focal plane of the beam. The polarization of the incident $\mathrm{THz}$ wave is parallel to the detector antenna. $\mathbf{c}$ The THz pulse waveform measured in the center of the focal spot; $t_{1}$ and $t_{2}$ indicate the time delay for images (b) and Fig. 4(a) respectively.

image shows the field pattern at $t=t_{1}$ (Fig. 3c). The field pattern evolves and the fringes move away from the center as time progresses. The fringes extend up to several hundred microns away from the focal point predominantly along the $x$-axis, the direction of the incident beam polarization. They are moving away from the center and decay as a cylindrically diverging wave, as it was shown in our recent study [16].

The image in Fig. $3 b$ shows that the surface waves propagating in the $+x$ and $-x$ directions have the same phase. However the anti-symmetric nature of the $E_{z}$ field predicts that the left side and the right side SPP waves should have opposite polarities and the $E^{S P P}$ pattern is expected to be anti-symmetric (along the $x$-axis). The change in the detected field polarity occurs due to the coupling of the wave into the probe. According to Eq. 2, the sign of the detected signal depends on the direction of the surface wave propagation. Because the wave vector projection on the $x$-axis has opposite signs on the left side and the right side, the experimental image exhibits the opposite symmetry compared to the actual $E^{S P P}$ pattern.

The $E^{S P P}$ field on the surface in principle can be reconstructed by integration of the detected spatial traces, provided that the relationship between the detected signal and the original field is described by the differential operators in Eq. 2. Some care must be taken when performing the integration because of the contribution due to the incident field $E_{\text {inc }}$, described by the last term in Eq. 2. This term can introduce an offset during the integration. The presence of the incident field in the image can be avoided in some cases. For example, if the dipole antenna orientation in the probe (described by the vector $\vec{a}$ ) is perpendicular to the incident field $\left(\vec{E}_{\text {inc }}(x, y, t) \cdot \vec{a}=0\right)$, the detected signal $i_{A}$ is entirely due to the SPP wave. In practice however it's difficult to avoid the contribution due to the incident field completely.

The field reconstruction method is illustrated in Fig. 4. We start with a near-field image recorded for the case of the incident beam polarized in the $y$-direction and the probe antenna aligned in the $x$-direction (Fig. 4a). The image shows the SPP wave as a 'spiral' pattern instead of a series of concentric fringes in Fig. $3 \mathrm{~b}$ and the central dark spot disappears. The original $E^{S P P}$ pattern is reconstructed in Fig. $4 \mathrm{~b}$ by integration of the spatial scan traces of 

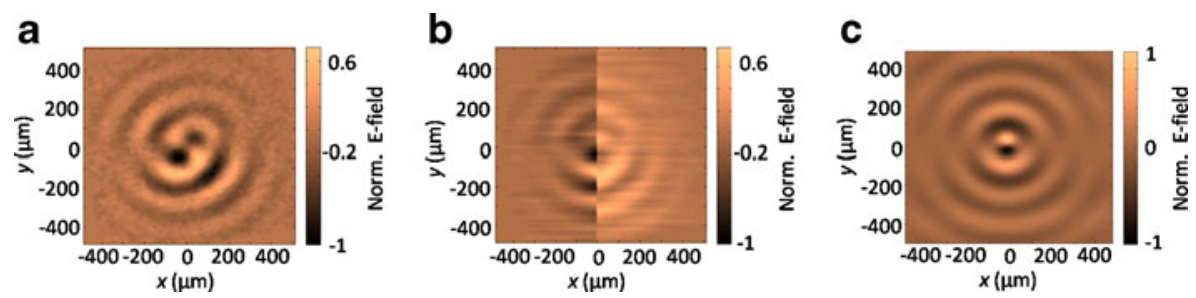

Fig. 4 a An image measured by the near-field probe ( $d=20$ micron) in the focal plane of the focused THz beam for the incident polarization orthogonal to the detector antenna. b A surface plasmon wave image reconstructed from the measured image in (a) by numerical integration. c Theoretical distribution of the surface plasmon field due to the focused $\mathrm{THz}$ beam excitation.

Fig. 4a. The orthogonal orientation of the antenna with respect to the incident field $E_{\text {inc }}$ reduces the offset significantly. In addition, the integration is performed separately on the left side and the right side of the image, starting from the image boundaries. In this case, the offset accumulated mostly in the center of the image does not affect the rest of the image. Although the merging line for two integral regions next to $x=0$ is visibly present, the reconstructed image reveals the pattern of the expected SPP wave shown in Fig. 4c [16, $25]$.

\subsection{Surface waves generated at metallic edges}

Before discussing SPP waves in complex metallic structures, it is insightful to consider how a surface wave forms on a single metallic edge. The edge can serve as the wave source because the incident field can induce a highly localized oscillating charge distribution, which in its turn starts the surface wave. To study surface waves produced at metallic edges we chose a metallic pattern of a large bow-tie antenna. The sample is fabricated by evaporating an opaque, $300 \mathrm{~nm}$ thick gold film on an InP substrate. The bow-tie antenna patch is approximately 10 times larger than the wavelength and the angle between the metallic edges and the polarization of the incident THz field is 60 degrees (Fig. 5a). The large flare angle ensures efficient excitation of the SPP wave while the large size allows observing the entire $\mathrm{THz}$ pulse within the antenna patch. In the experimental setup, the bow-tie structure is illuminated by an unfocused THz beam with a profile similar to Fig. 1c. Images are recorded using a near-field probe with a $50 \mu \mathrm{m}$ aperture. The probe is scanned within about $20 \mu \mathrm{m}$ from the sample surface.

When the $\mathrm{THz}$ pulse reaches the sample, surface waves are excited at the edges of the bow-tie pattern. The waves then propagate on the surface of the metallic layer away from the edges. Two-dimensional images of the sample are taken at two different moments in time, $t_{1}$ and $t_{2}$, indicated by dashed lines in the space-time map in Fig. 5b. These moments correspond to a positive peak and a negative peak of the electric field in the leading region of the incident $\mathrm{THz}$ pulse and they are separated by an interval of one half of the wave cycle. The corresponding images are shown in Fig. 5c, d. They illustrate that the SPP wave front is moving away from the edge. The surface wave propagation is particularly clear in the space-time map in Fig. 5b, where the SPP waves propagating away from the bow-tie antenna inner edges appear as an $\Lambda$-shape pattern. Such patterns are common for surface plasmon waves [16]; they can be used to identify and analyze SPP waves. For example, the phase velocity can be calculated from the slope of the $\Lambda$-shape pattern in Fig. 5b. Taking into account that the map is measured along a line that forms an angle of 30 degrees with 

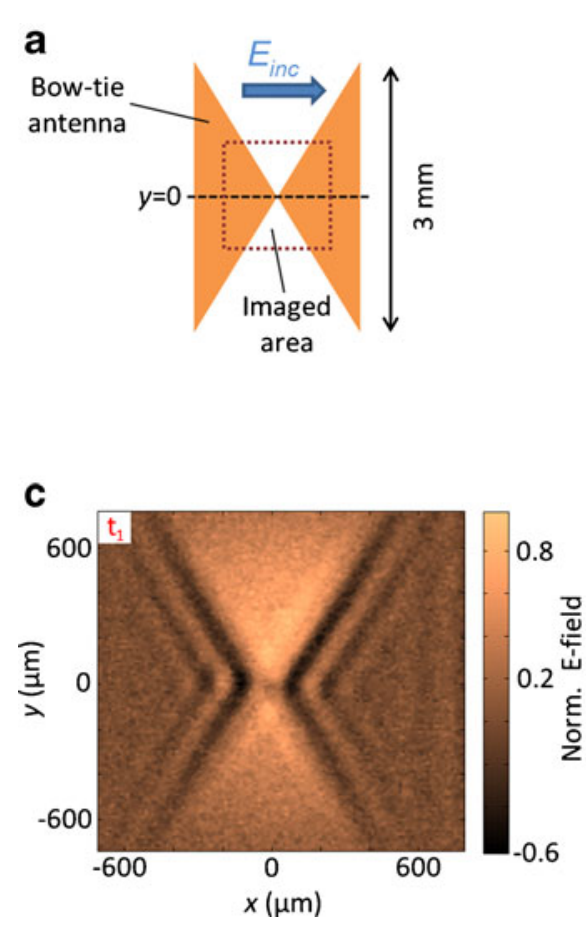
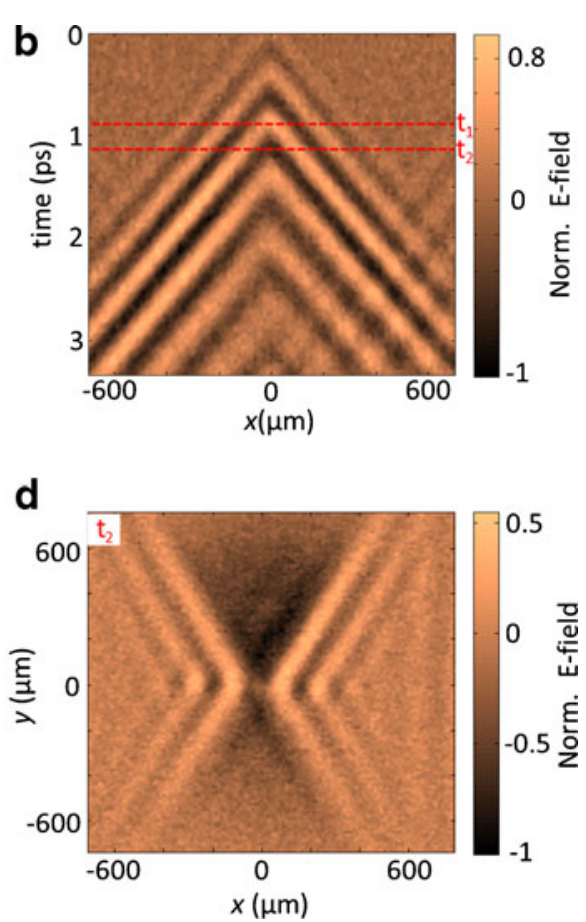

Fig. 5 a A schematic diagram of a large bow-tie antenna. b A space-time map measured along a horizontal line $(y=0)$ through the antenna center. $\mathbf{c}, \mathbf{d}$ THz near-field images of the structure in (a) measured with the 50 micron aperture probe. The images are recorded at $t=t_{1}$ and $t_{2}$ indicated by red dashed lines in the spacetime map.

the SPP wave vector, the phase velocity is found to be $3.0 \pm 0.1 \cdot 10^{8} \mathrm{~m} / \mathrm{s}$ matching the expected value within the measurement error.

As in the case of the surface wave excited by a focused beam, the differential nature of the detected signal is reflected in the image symmetry. For the $x$-polarized incident $\mathrm{THz}$ field, the $E_{z}$ components of the SPP wave on the left side and the right side of the metallic structure are expected to have opposite polarities. The observed SPP wave pattern however must be symmetric due to different signs of the wave vector projections on the detector antenna vector $\vec{a}$. The $2 \mathrm{D}$ images and the space-time map reflect this symmetry and confirm the coupling mechanism described by Eq. 2 .

The images also show clearly that the electromagnetic field can exist behind opaque metallic structures because THz SPP waves can form at the metallic edges and propagate into the shadow region. The understanding of the SPP wave formation at the edges is vital for analysis of complex metallic samples, in which the dimensions are comparable or smaller than the wavelength, such as antennas, transmission lines and resonant structures employed in metamaterials.

\subsection{Surface waves on $\mathrm{THz}$ devices: bow-tie antenna}

As the final example we consider a small resonant structure, a bow-tie antenna with dimensions comparable to the wavelength size. In such a sample we need to consider reflection and interference of the SPP waves to explain the observed patterns in near-field 

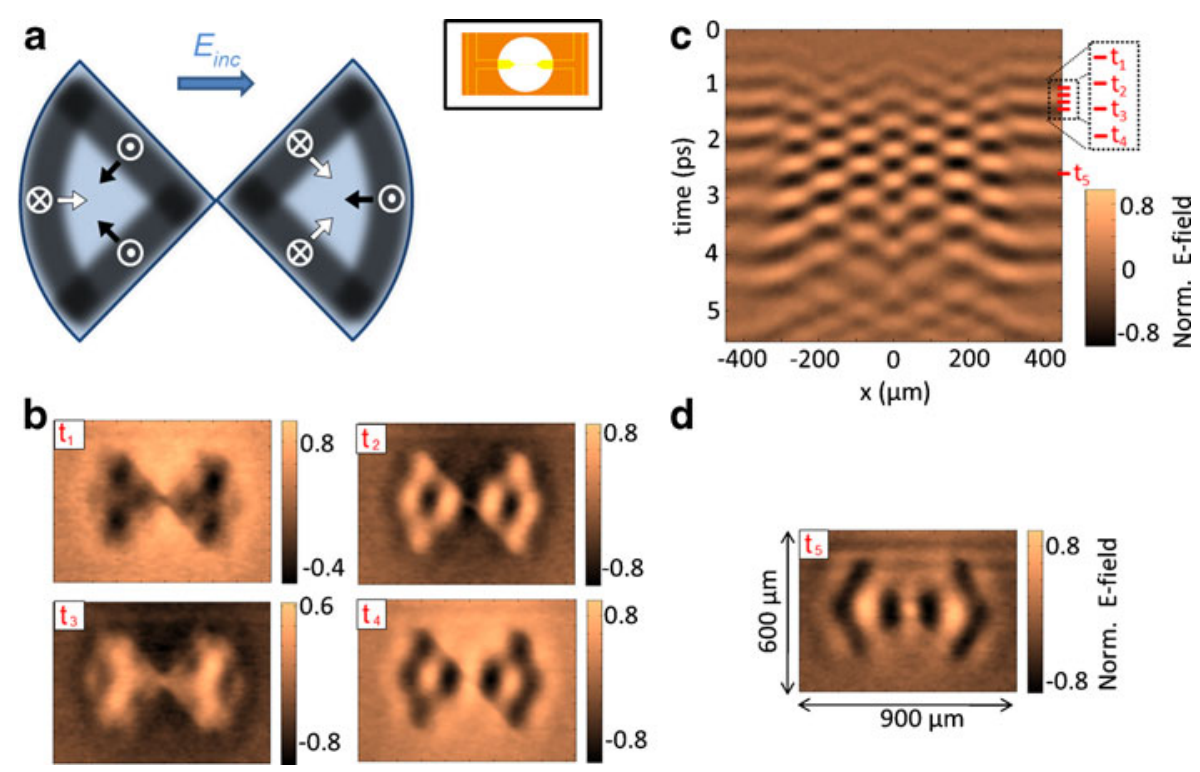

Fig. 6 a A schematic diagram of a small bow-tie antenna and the surface waves excited at the metallic edges for the incident field polarized along the $x$-axis. The inset shows the detector orientation. b THz near-field images of the structure measured with the 20 micron aperture probe. The images are recorded at $t=t_{1}, t_{2}, t_{3}$ and $t_{4}$ indicated by red dashed lines in the space-time map. c A space-time map measured along a horizontal line $(y=0)$ through the antenna center. $\mathbf{d}$ A near-field image measured at $t=t_{5}$.

images. The antenna structure is schematically shown in Fig. 6a. It is imaged in a similar configuration as the larger bow-tie antenna. A $20 \mu \mathrm{m}$ aperture probe is used in this experiment to improve spatial resolution.

According to Eq. 2, the polarity of the detected signal depends on both the direction of the wave vector and the direction of the SPP field. We introduce the following schematic notations to display the direction of the SPP wave vector and the electric field in Fig. 6a: the direction of the normal electric field $E^{S P P}$ is shown by a circle with a dot or a cross, which correspond to the $E^{S P P}$ field pointing in or out of the sample plane. The direction of the SPP wave vector is shown by arrows, whereas the arrow color indicates the sign of the scalar product $\vec{k}_{s p p} \cdot \vec{a}$ (i.e. a white (black) arrow corresponds to a positive (negative) value of the product). The polarity of the expected signal is schematically illustrated by the color tone: bright regions correspond to the positive signal and dark regions correspond to the negative signal. Figure 6 a schematically illustrates the surface waves excited at all edges of the bow-tie structure illuminated by the $x$-polarized THz pulse. It shows that constructive "interference" of the surface waves is expected near the bow-tie corners.

A series of four experimental near-field images are shown in Fig. 6b. Although individual waves originating from the antenna edges are not clearly resolved, the structure of the observed patterns resembles the interference pattern in Fig. 6a. The images represent the evolution of the SPP pattern during one complete cycle of the THz field (Fig. 6c). All four images display the superposition of the surface waves originating from the bow-tie antenna edges. Since the SPP waves can reflect from the metallic boundaries, the surface wave resonance can build up over time. The interference pattern therefore changes slightly as shown in Fig. 6d. The outer edges of the bow-tie antenna play the main role in building the resonance because they act as focusing reflectors that force the surface waves to 


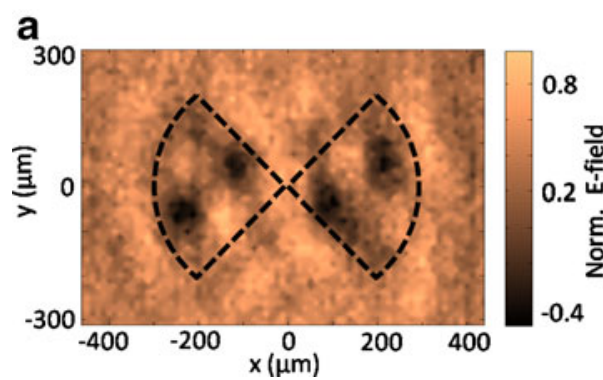

b

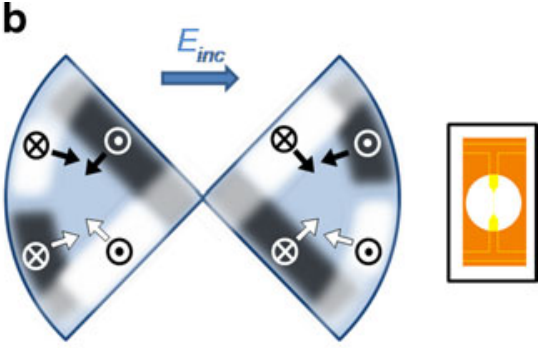

Fig. 7 a THz near-field image of a small bow-tie antenna measured with the 20 micron aperture probe. b Schematic diagram of the sample and the surface waves excited at the metallic edges for the incident field polarized along the $\mathrm{x}$-axis. The inset shows the detector orientation.

propagate from these edges directly to the antenna center. A standing wave pattern develops after a couple of picoseconds (Fig. 6c, d).

Finally, to illustrate the differential nature of the detected signal, we consider a near-field image recorded with the detector antenna oriented perpendicular to both the incident $\mathrm{THz}$ field and the axis of the bow-tie antenna (Fig. 7a). In the experiment, the detector is kept stationary while the sample-waveguide assembly and the polarization of the incident $\mathrm{THz}$ beam are rotated by $90 \mathrm{deg}$. The same configuration can also be realized by rotating the near-field detector by $90 \mathrm{deg}$. The gradient term in Eq. 2 now contains only the $y$-coordinate derivative and the second term due to the incident $\mathrm{THz}$ field disappears. We can predict the SPP pattern for this configuration using the same notations as in Fig. 6a. Although the excitation of the bow-tie antenna and the $E^{S P P}$ field pattern remain the same as in Fig. 6, the orientation of the dipole antenna vector $\vec{a}$ is now along the $y$-direction. As a consequence, the color of the arrows is different on the top and bottom sides of the bow-tie and the detected pattern has the reversed mirror symmetry with respect to the $x$-axis passing through the bow-tie center. The expected near-field image pattern is illustrated in the schematic diagram of Fig. 7b. Another consequence of Eq. 2 is the disappearance of the SPP waves along the $x$-axis. The wave becomes invisible because the wave vector $k_{s p p}$ is perpendicular to detector antenna vector $\vec{a}$. These features are reproduced precisely in the near-field image in Fig. 7a: the pattern displays the expected symmetry and there is no detected field along the $x$-axis.

\section{Conclusion}

The examples discussed above show that $\mathrm{THz}$ surface waves play an important role in image formation in near-field scanning probe microscopy. $\mathrm{THz}$ surface waves can be excited at the metallic edges of samples as well as on the surface of the near-field probe itself. The sensitivity of the integrated sub-wavelength aperture probe to the $\mathrm{THz}$ surface plasmon waves opens exciting opportunities for experimental investigations of SPP phenomena. The example of the bow-tie antenna demonstrates the capability of the probe to investigate resonant SPP effects in THz devices. Supplemented with the time-domain analysis, $\mathrm{THz}$ near-field microscopy can provide a vital insight into the SPP role in properties of $\mathrm{THz}$ devices, including metamaterials, waveguides and antennas, and it can help to develop novel $\mathrm{THz}$ devices.

The understanding of the surface wave coupling into the probe reveals the nature of several unusual imaging effects that have been observed in earlier near-field microscopy 
studies. The polarization dependent resolution in spatial resolution edge tests [26] can be unambiguously explained by the formation of the surface wave at the edge. 'Bright' features behind opaque metallic patterns [15] are caused by interference of the surface waves excited at the metallic edges. Surface waves excited on the near-field probe surface can also produce imaging artefacts. For example, images of modes in metallic $\mathrm{THz}$ waveguides can display fringes near the metallic waveguide walls [21]. The SPP nature of these effects becomes clear with the understanding of the SPP wave coupling into the probe.

In this work, we provide several examples, which confirm that the surface wave field detected by the integrated sub-wavelength aperture near-field probe corresponds to a spatial derivative of the surface field distribution. Although the relationship is not direct, the nearfield images nevertheless display the SPP waves. The spatial derivative nature of the detected signal however requires further analysis for correct image interpretation. This study provides a framework for the analysis and illustrates several analytical methods. In special cases, the framework allows reconstruction of the original $E^{S P P}$ field distribution by applying the numerical integration transformation to near-field images.

The coupling mechanism is now understood, however further quantitative studies are needed to improve the near-field image analysis. Among the most important is the question of the probe frequency response for the SPP waves and its dependence on the aperture size and shape. Addressing this question will allow quantitative spectroscopic analysis of the SPP phenomena. The effect of the probe on the SPP waves propagating on the sample is another important aspect that needs to be investigated.

Acknowledgement This work was supported by the Royal Society [grant number UF080745] and the Engineering and Physical Sciences Research Council [grant number EP/G033870/1].

\section{References}

1. K. Wang and D. M. Mittleman, "Metal wires for terahertz waveguiding", Nature 432, 376-379 (2004).

2. H. Zhan, R. Mendis, and D. Mittleman, "Superfocusing terahertz waves below $\lambda / 250$ using plasmonic parallel-plate waveguides", Opt. Express 18, 9643-9650 (2010).

3. M. Gong, T. Jeon, and D. Grischkowsky, "THz surface wave collapse on coated metal surfaces", Opt. Express 17, 17088-17101 (2009).

4. J. Saxler. J. G. Rivas, C. Janke, H. P. M. Pellemans, P. H. Bolivar, and H. Kurz, "Time-domain measurements of surface plasmon polaritons in the Terahertz frequency range", Phys. Rev. B. 69, 1554271-1554274 (2004).

5. F. Miyamaru, et al., "Terahertz surface wave resonant sensor with a metallic hole array," Opt. Lett. 31, 1118-1120 (2006).

6. S. Pandey, G. Kumar, and A. Nahata, "Slot waveguide-based splitters for broadband terahertz radiation", Opt. Express 18, 23466-23471 (2010).

7. M. Theuer, R. Beigang, and D. Grischkowsky, "Highly sensitive terahertz measurement of layer thickness using a two-cylinder waveguide sensor", Appl. Phys. Lett. 97, 071106 (2010).

8. N. C. J. van der Valk and P. C. M. Planken, "Effect of a dielectric coating on terahertz surface plasmon polaritons on metal wires", Appl. Phys. Lett. 87, 071106 (2005)

9. W. Barnes, A. Dereux, and T. Ebbesen, "Surface plasmon subwavelength optics", Nature 424, 824-830 (2003).

10. A. Bitzer and M. Walther, "Terahertz near-field imaging of metallic subwavelength holes and hole arrays", Appl. Phys. Lett. 92, 231101-231103 (2008).

11. J. R. Knab, A. J. L. Adam, M. Nagel, and E. Shaner, M. A. Seo, D. S. Kim, and P. C. M. Planken, "Terahertz Near-Field Vectorial Imaging of Subwavelength Apertures and Aperture Arrays", Opt. Express 17, 15072-15086 (2009).

12. A. Bitzer, A. Ortner, H. Merbold, T. Feurer, and M. Walther, "Terahertz near-field microscopy of complementary planar metamaterials: Babinet's principle," Opt. Express 19, 2537-2545 (2011). 
13. N. C. J. van der Valk, P. C. M. Planken, "Electro-optic detection of sub-wavelength terahertz spot sizes in the near-field of a metal tip," Appl. Phys. Lett. 81, 1558-1560 (2002)

14. A. J. Huber, F. Keilmann, J. Wittborn, J. Aizpurua, and R. Hillenbrand, "Terahertz Near-Field Nanoscopy of Mobile Carriers in Single Semiconductor Nanodevices", Nano Lett. 8, 3766-3770 (2008).

15. O. Mitrofanov, M. Lee, J. W. P. Hsu, I. Brener, R. Harel, J. F. Federici, J. D. Wynn, L. N. Pfeiffer, and K. W. West, "Collection-Mode Near-Field Imaging with 0.5-THz Pulses", IEEE J. Sel. Top. Quantum Electron. 7, 600-607 (2001).

16. R. Mueckstein and O. Mitrofanov, "Imaging of terahertz surface plasmon waves excited on a gold surface by a focused beam," Opt. Express 19, 3212-3217 (2011).

17. O. Mitrofanov, L. N. Pfeiffer, and K. W. West, "Generation of low-frequency components due to phaseamplitude modulation of subcycle far-infrared pulses in near-field diffraction", Appl. Phys. Lett. 81, 1579 (2002).

18. O. Mitrofanov, I. Brener, M. C. Wanke, R. R. Ruel, J. D. Wynn, A. J. Bruce, and J. Federici, "Near-field microscope probe for far infrared time domain measurements", Appl. Phys. Lett. 77, 591 (2000).

19. B. Bowden, J. A. Harrington and O. Mitrofanov, "Low-loss modes in hollow metallic waveguides with dielectric coatings", Appl. Phys. Lett., 93, 181104 (2008).

20. B. Bowden, J. A. Harrington and O. Mitrofanov, "Fabrication of terahertz hollow-glass metallic waveguides with inner dielectric coatings", J. App. Phys. 104, 093110 (2008).

21. O. Mitrofanov, T. Tan, P. R. Mark, B. Bowden, and J. A. Harrington, "Waveguide mode imaging and dispersion analysis with terahertz near-field microscopy", Appl. Phys. Lett. 94, 1711041-1711043 (2009).

22. O. Mitrofanov and J. A. Harrington, "Dielectric-lined cylindrical metallic THz waveguides: mode structure and dispersion," Opt. Express 18, 1898-1903 (2010).

23. A. Bouhelier, F. Ignatovich, A. Bruyant, C. Huang, G. Colas des Francs, J.-C. Weeber, A. Dereux, G.P. Wiederrecht, and L. Novotny, "Surface plasmon interference excited by tightly focused laser beams", Opt. Lett. 32, 2535-2537 (2007).

24. H. Kano, S. Mizuguchi, and S. Kawata, "Excitation of surface-plasmon polaritons by a focused laser beam", J. Opt. Soc. Am. B 15, 1381-1386 (1998).

25. L. Novotny and B. Hecht, Principles of Nano-Optics (Cambridge University Press, 2006).

26. O.Mitrofanov et al. "Terahertz near-field microscopy based on a collection mode detector", Appl. Phys. Lett., 77, 3496 (2000). 